\title{
Inhibition of Wntless/GPR177 suppresses gastric tumorigenesis
}

\author{
Jaesung Seo,"\#, Hyun Jung Kee ${ }^{2, \#}$, Hye Ji Choi ${ }^{1,2}$, Jae Eun Lee ${ }^{2}$, Soo-Yeon Park ${ }^{1}$, Seung-Hyun Lee ${ }^{1}$, Mi-Hyeon Jeong ${ }^{1}$, \\ Garam Guk ${ }^{1}$, SooYeon Lee ${ }^{1}$, Kyung-Chul Choi ${ }^{3}$, Yoon Young Choi ${ }^{2}$, Hyunki Kim ${ }^{4}$, Sung Hoon Noh ${ }^{2}$, Ho-Geun Yoon ${ }^{1, *} \mathcal{E}^{*}$ \\ Jae-Ho Cheong, ${ }^{1,2, *}$ \\ ${ }^{1}$ Department of Biochemistry and Molecular Biology, Brain Korea 21 PLUS Project for Medical Sciences, Yonsei University College of \\ Medicine, Seoul 03722, ${ }^{2}$ Department of Surgery, Yonsei University College of Medicine, Seoul 03722, ${ }^{3}$ Department of Biomedical Sciences, \\ University of Ulsan College of Medicine, Seoul 05505, ${ }^{4}$ Department of Pathology, Yonsei University College of Medicine, Seoul 03722, \\ Korea
}

\begin{abstract}
Wntless/GPR177 functions as WNT ligand carrier protein and activator of WNT/ $\beta$-catenin signaling, however, its molecular role in gastric cancer (GC) has remained elusive. We investigated the role of GPR177 in gastric tumorigenesis and provided the therapeutic potential of a clinical development of anti-GPR177 monoclonal antibodies. GPR177 mRNA expression was assessed in GC transcriptome data sets (GSE15459, $n$ = 184; GSE66229, $n=300$ ); protein expression was assessed in independent patient tumor tissues (Yonsei TMA, $n=909$ ). GPR177 expression were associated with unfavorable prognosis [log-rank test, GSE15459 ( $P=0.00736)$, GSE66229 $(P=$ 0.0142), and Yonsei TMA ( $P=0.0334)]$ and identified as an independent risk predictor of clinical outcomes: GSE15459 [hazard ratio (HR) 1.731 (95\% confidence interval; $\mathrm{Cl}$; 1.1032.715), $P=0.017$ ], GSE66229 [HR 1.54 (95\% Cl, 1.10-2.151), $\mathrm{P}=0.011]$, and Yonsei TMA [HR $1.254(95 \% \mathrm{Cl}$, 1.0491.500), $P=0.013$ ]. Either antibody treatment or GPR177 knockdown suppressed proliferation of GC cells and sensitized cells to apoptosis. And also inhibition of GPR177 suppresses in vitro and in vivo tumorogenesis in GC cells and inhibits WNT/ $\beta$-catenin signaling. Finally, targeting and inhibition of GPR177 with antibody suppressed tumorigenesis in PDX model. Together, these results suggest GPR177 as a novel candidate for prognostic marker as well as a promising target for treatment of GC patients. [BMB Reports 2018; 51(5): 255-260]
\end{abstract}

*Corresponding authors. Ho-Geun Yoon, Tel: +82-2-2228-1684; Fax: +82-2-312-5041; E-mail: yhgeun@yuhs.ac; Jae-Ho Cheong, Tel: +82-2-2228-2094; Fax: +82-2-313-8289; E-mail: jhcheong@ yuhs.ac

${ }^{\#}$ These authors contributed equally to this work.

https://doi.org/10.5483/BMBRep.2018.51.5.046

Received 26 February 2018, Revised 13 March 2018, Accepted 20 March 2018

Keywords: Gastric cancer, GPR177, Monoclonal antibody, PDX, WNT signaling

\section{INTRODUCTION}

Gastric cancer (GC) is one of the most common malignancies and the third leading cause of cancer death worldwide (1). Many studies have attempted to elucidate the molecular mechanisms underlying gastric tumorigenesis and develop novel molecular targeted therapeutics $(2,3)$. Recently, anti-HER2 receptor and anti-VEGFR2 receptor antibodies were developed and approved as targeted therapy for GC patients (4-6); however, identification and validation of more candidate therapeutic targets are still required (7).

Dysregulation of $\mathrm{WNT} / \beta$-catenin signaling is commonly observed in cancer, including GC (8-10). When WNT ligands are secreted and interact with their cognate receptors, $\beta$-catenin-dependent or -independent signaling cascades are transduced, resulting in cancer progression. In the absence of WNT ligand, $\beta$-catenin is maintained at a low level through degradation by the $\beta$-catenin destruction complex. Secreted WNT ligand binds to FZD-LRP receptors, triggering the WNT/ $\beta$-catenin axis. As a consequence, AXIN complexes with DVL which inhibits $\beta$ - TrCP to degrade beta catenin, resulting in cytoplasmic accumulation of $\beta$-catenin, which then translocates into the nucleus where it binds to the TCF/LEF transcription complex, activating target genes transcription (11). In many types of cancer, mutations in CTNNB1, AXIN, and APC cause hyperactivation of WNT/ $\beta$-catenin signaling, resulting in cancer progression even in the absence of WNT ligand stimulation. Therefore, various small molecules and antibodies are under development as cancer therapeutics. A recent report suggests that blocking of WNT secretion may be a useful means of treating colorectal cancer $(12,13)$.

WNTs are secreted as glycosylated/lipid-modified proteins, mediated by the seven-pass transmembrane protein Wntless/GPR177 (14-16). GPR177 localizes to compartments of the secretory pathway, including the ER, Golgi apparatus, endosomes, and plasma membrane. GPR177 is overexpressed in several cancer types, and GPR177 knockdown reduced cancer formation in a WNT-dependent manner (17). Growth

ISSN: 1976-670X (electronic edition)

Copyright (c) 2018 by the The Korean Society for Biochemistry and Molecular Biology

(c) This is an open-access article distributed under the terms of the Creative Commons Attribution Non-Commercial License (http://creativecommons.org/licenses/by-nc/4.0) which permits unrestricted non-commercial use, distribution, and reproduction in any medium, provided the original work is properly cited. 
of colon cancer cells harboring the $\beta$-catenin active mutant or APC mutant was inhibited in a GPR177-dependent manner. Thus, these studies suggest that blockade of GPR177 function may be a promising therapeutic target $(13,18-20)$.

Here, we demonstrate that overexpression of GPR177 mRNA and protein correlates with poor prognosis of GC patients. WNT secretion and cell proliferation were inhibited by either anti-GPR177 monoclonal antibody treatment or GPR177 knockdown. Moreover, anti-GPR177 antibody exhibited anticancer efficacy in mouse and patient-derived xenograft (PDX) models.

\section{RESULTS}

\section{GPR177 overexpression in GC is correlated with poor survival}

To investigate the clinical significance of GPR177 in GC, we analyzed publicly available tumor transcriptome data sets and found that GPR177 mRNA level strongly correlated with poor overall survival in patients with GC (Fig. $1 \mathrm{~A}$ and B). To evaluate whether GPR177 mRNA level correlates with protein level while maintaining the clinical implications, we analyzed GPR177 protein expression in tissue microarrays (TMAs) prepared using tissue of GC patients $(\mathrm{n}=909)$ and a monoclonal antibody generated for this study (Fig. 1C and Supplementary Table 1). Patient age and sex, tumor histology, Lauren classification, and pathological tumor-node-metastasis
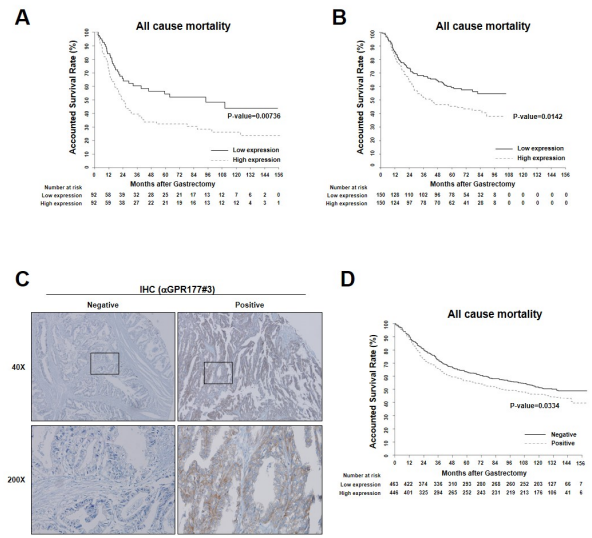

Fig. 1. Clinical impact of GPR177 in gastric cancer (GC) patients (A-D) GPR177 expression correlates with poor prognosis in human GC patients. (A and B) Correlation of GPR177 (WLS) mRNA expression with overall survival for patients $(A, n=184$; $\mathrm{B}, \mathrm{n}=300$ ). Solid and broken lines denote patients whose tumors expressed lower and higher levels, respectively, than the median value. (C) Micrographs of immunohistochemical staining of GPR177 in human GC tumor samples. Representative images of negative (left) and positive (right) staining. Scale bar, $100 \mu \mathrm{m}$. (D) Kaplan-Meier analysis of overall survival in a data set of 909 GC samples used in immunohistochemical analysis and classified as having positive (broken line) or negative (solid line) GPR177 expression.
(TNM) stage were analyzed as clinicopathological parameters in relation to GPR177 expression level. Intriguingly, all clinical and histological parameters were comparable between GPR177-negative $(n=463)$ and GPR177-positive $(n=446)$ GC patients, with the exception of tumor histology (Supplementary Table 1). In general, undifferentiated GC histology would correlate with unfavorable clinical outcomes; however, GPR177-positive tumors were significantly correlated with more highly differentiated histological subtypes, signifying that GPR177 expression may be independently associated with poor clinical outcomes. Indeed, positive GPR177 protein expression correlated significantly with poorer prognosis of GC patients (Fig. 1D). Furthermore, GPR177 expression at both the mRNA and protein levels was identified as an independent risk predictor of clinical outcome of GC patients even after adjusting the covariates as Lauren and TNM stages (Supplementary Table 2). Together, the clinical data suggest that GPR177 expression may be a reliable and legitimate predictor of unfavorable clinical outcome of GC
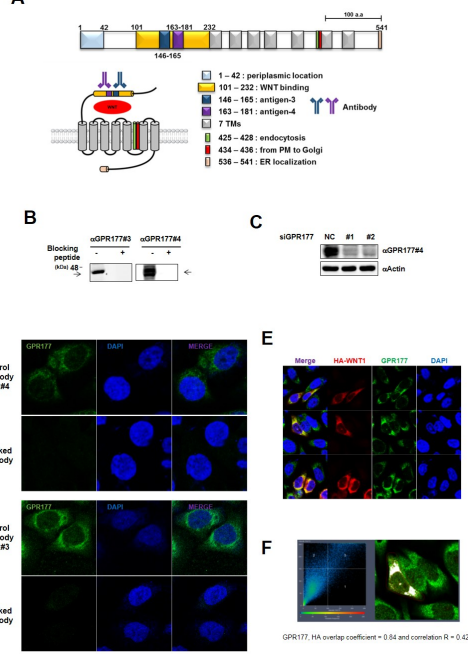

Fig. 2. GPR177 co-localizes with WNT1 in GC cell lines. (A) Schematic diagram of GPR177 structure and function. Upper: linear representation of GPR177. Lower: GPR177, with WNT cargo, spanning plasma membrane. The seven transmembrane domains consist of amino acids 233-252, 265-287, 302-319, 332-354, 379-401, 434-456, and 471-493. (B) AGS cell lysates were loaded and analyzed with GPR177 antibody in western blot with or without blocking peptide. Endogenous GPR177 was not detected in blocking peptide treated condition. (C) Validation of siRNA specific for GPR177. AGS cells were were transiently transfected with siRNA as indicated. Full-length blots merged with protein size markers are presented in Supplementary Fig. 3. (D) AGS cells were stained with GPR177 antibody with or without blocking peptide. (E) AGS cells were transiently expressed with HA-WNT1 and stained with anti-HA (red) and anti-GPR177 (green). Overexpression of WNT1 induced GPR177 expression. (F) WNT1 and GPR177 were co-localized. Images were processed using Carl Zeiss ZEN software. 
patients, evidence that further investigation of molecular mechanisms in GC tumorigenesis is warranted.

Both GPR177-mediated WNT secretion and proliferation of GC cells are inhibited by anti-GPR177 monoclonal antibody GPR177 binds cytoplasmic WNT ligand and secretes it into the extracellular milieu. GPR177 domains and their functions are described in Fig. 2A. To analyze its biological function in GC cell lines, we raised monoclonal antibodies that recognize the WNT-binding motif of GPR177. Antibodies were raised against each of five antigens. The monoclonal antibodies raised against antigen 3 (antibody \#3) and antigen 4 (antibody \#4) were selected and specificity was verified by western analysis with GPR177 blocking peptide and knockdown AGS cells (Fig. 2B and C). And also, GPR177 localization was assessed by immnunocytochemistry with generated antibody and the specificity for the antibody was confirmed by blocking peptide (Fig. 2D). Notably, GPR177 was co-localized and was increased with WNT1 expression as precedent report (Fig. 2E and F) (14).

Considering heterogeneity of GC, we evaluated GPR177 expression levels in several GC cell lines using qPCR. GPR177 expression levels showed a certain degree of variation across cell lines which are AGS, MKN45, YCC6, YCC7, YCC16 and 293FT as positive control for WNT-secreting cells (Fig. 3A). Next, we examined the possibility that GPR177 is required for WNT secretion from GC cells. GC cells transiently expressing HA-tagged WNT1 were cultured with or without anti-GPR177 antibody (Fig. 3B). Levels of WNT ligand in medium were assessed by anti-HA ELISA. All cell lines secreted WNT ligand, and secretion was inhibited by treatment with anti-GPR177 antibody. Based on these observations, we found that anti-GPR177 antibody efficiently blocks a WNT-secretory function of GPR177. We also observed that viability of WNT-secreting cells was suppressed by anti-GPR177 antibody in a concentration-dependent manner (Fig. 3C and D). Whereas WNT overexpression increased the migration of AGS cells compared with control cells, and this effect was reversed by monoclonal antibody treatment (Fig. 3E). Similar results were observed using a colony-forming assay (Fig. 3F). Since we observed the inhibitory activity of anti-GPR177 antibody on the proliferation gastric cancer cells, we next assessed the change of apoptosis in gastric cancer cells with or without anti-GPR177. Anti-GPR177 antibody treatment dramatically increased the annexin V-positive cells in both YCC6 and MKN45 cells, which were again verified with knocking-down of GPR177 (Fig. 3G).

It has been reported that MKN74 cells are capable of brain metastasis upon injection into mouse heart, and the brain metastatic (NI3-3) cells have been established in cell culture (21). NI3-3 cells are sensitive to anti-GPR177 antibody treatment compared with the parental MKN74 cells (Fig. $3 \mathrm{H}$ ) and expressed high levels of GPR177 (WLS), AXIN2, and cyclin D1 (CCND1) mRNA (Fig. 3l) which are the main targets

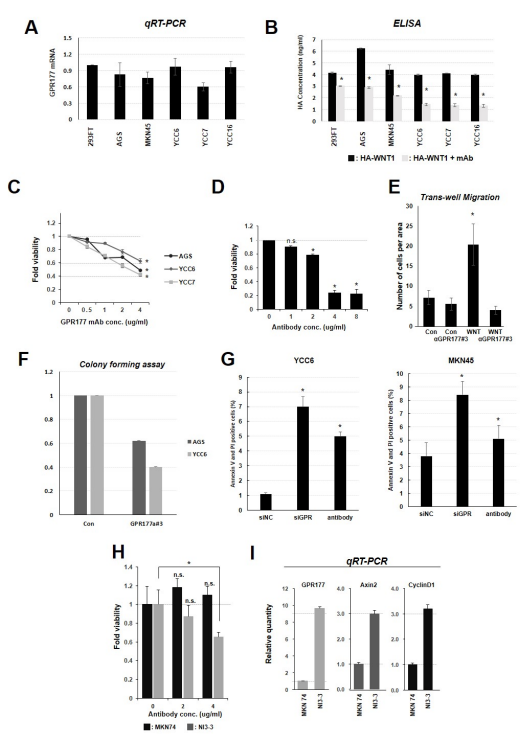

Fig. 3. GPR177 monoclonal antibody treatment inhibits WNTsecretion and viability of gastric cancer cells. (A) GPR177 mRNA expression was measured by qPCR. GC cell lines (AGS, MKN25, YCC6, YCC7, and YCC16) and 293FT cells were collected and analyzed. GC cells showed expression levels similar to that of 293FT cells, (B) ELISA detection of secreted HA-WNT1. WNT secretion was reduced by monoclonal antibody treatment. $* \mathrm{P}<$ 0.05. (C) GPR177 monoclonal antibody treatment reduced cell viability. AGS, YCC6 and YCC7 were treated with GPR177 monoclonal antibody at the indicated concentration for $24 \mathrm{~h}$. Cells showed reduced viability with antibody treatment. (D) AGS cells were treated with monoclonal antibody at the indicated concentrations and for $24 \mathrm{hr}$ to determine lethal dose (LD)50. Viability was assessed by MTT assay. ${ }^{*} P<0.05$. (E) Monoclonal antibody treatment reduced cell migration. AGS cells transiently overexpressing WNT and control cells were seeded on transwell plates, and migrated cells were counted. GPR177 monoclonal antibody suppressed cell migration (concentration $1 \mu \mathrm{g} / \mathrm{ml}$ ). ${ }^{* P}<$ 0.05. (F) WNT-secreting AGS and YCC6 cells were seeded at $60-\mathrm{mm}$ dish and treated with monoclonal antibody. Colonies were fixed and stained with crystal violet and counted under microscope. ${ }^{*} \mathrm{P}<0.05$. (G) YCC6 and MKN45 cells were treated with 5 pmol GPR177 siRNA for $48 \mathrm{~h}$ or antibody. Apoptosis was assayed by FACS. (H) MKN74 and NI3-3 cells were treated with 2 or $4 \mu \mathrm{g} / \mathrm{ml}$ monoclonal antibody for $24 \mathrm{~h}$. Suppression was observed only in NI3-3 cells. ${ }^{* P}<0.05$. (I) qPCR analysis showed that WNT/ $\beta$-catenin signaling is upregulated in NI3-3 cells compared to MKN74 cells. GPR177 (WLS), AXIN2, and cyclin D1 (CCND1) mRNA levels were detected.

for the WNT/ $\beta$-catenin pathway. Taken together, WNT secretion and viability of gastric cancer cells are efficiently inhibited by treatment with anti-GPR177 monoclonal antibodies.

\section{Anti-GPR177 antibody treatment suppresses gastric tumorigenesis in vivo}

To evaluate in vivo efficacy of anti-GPR177 monoclonal antibodies, MKN45 cells were injected into nude mice $(n=5$ each). After two weeks of injection, anti-GPR177 antibodies 
were delivered intravenously every 2 days for 3 weeks, and tumor size was measured. Treatment with anti-GPR177 antibodies reduced tumor growth significantly (Fig. 4A). Upon

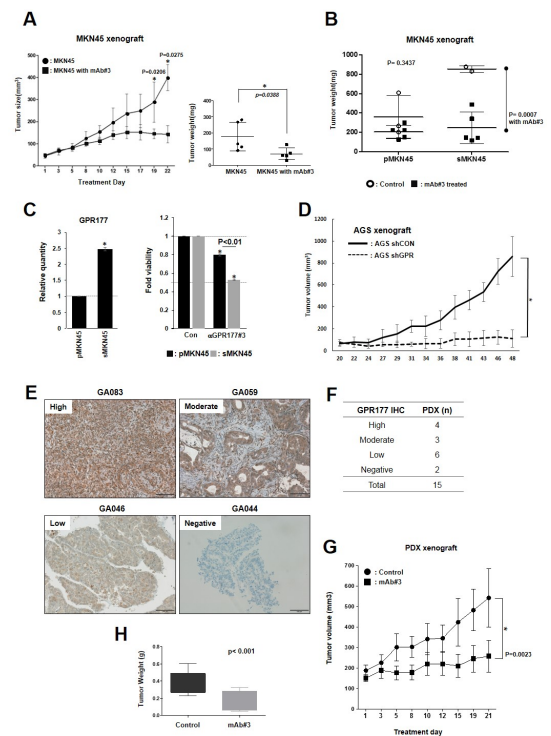

Fig. 4. GPR177 monoclonal antibody inhibits tumor growth in GC xenograft and human PDX model. (A) Nude BALB/C mice were injected subcutaneously with $10^{5}$ MKN45 cells. Two weeks later, mice were randomly divided into two groups of 10 mice each. One group was intravenously injected with anti-GPR177 monoclonal antibody \#3 ten times over 3 weeks, approximately every 2 days. Control group was injected with vehicle PBS. Tumor size was measured with calipers. At the end of anti-tumor efficacy test, the mice were scarified, and then tumors dissected and weighted. (B) Parental MKN45 (pMKN45) and metabolicstress-selected MKN45 (sMKN45) cells $\left(10^{5}\right)$ were subcutaneously injected into nude mice. Two weeks later, mice were randomly divided into two groups of 5 each in both cells. One group was intravenously injected with anti-GPR177 monoclonal antibody \#3 eight times over 3 weeks, and the other was treated with vehicle PBS. sMKN45 cells were also significantly inhibited by antibody treatment. $\mathrm{P}=0.0023$ for monoclonal antibody \#3 in tumor weight. (C) Metabolic stress induces GPR177 in sMKN45 cells. GPR177 mRNA levels in pMKN45 and sMKN45 cells were determined by qPCR. Monoclonal antibody treatment $(2 \mu \mathrm{g} / \mathrm{ml}$ for $24 \mathrm{~h})$ reduced sMKN45 cell viability to a greater extent than pMKN45 cell viability determined by MTT assay. ${ }^{*} P<0.05$. (D) Stable shRNA-mediated knockdown of GPR177 in AGS cells. Control cells were treated with non-targeting shRNA. Cells were subcutaneously injected into nude mice. Tumors were measured 2 weeks later with calipers every 2 days. ${ }^{*} \mathrm{P}<0.05$. (E) Representative cases of PDX models according to GPR177 expression levels. GPR177 expression was detected by immunohistochemistry using anti-GPR177 antibody \#3. (F) Summary of PDX models by GPR177 expressions. Cases were classified based on GPR177 expression level as high, moderate, low and negative expression. (G) GA083 tumors were transplanted to athymic nude mice and treated either with anti-GPR177 monoclonal antibody \#3 eight times over 3 weeks $(n=10)$ or with vehicle PBS $(n=10)$. $(H)$ Tumor weight of dissected tumors in $\mathrm{C}$ ). completion of experiments, tumors were removed from mice and further analyzed by western blotting. Intriguingly, we found that the WNT/ $\beta$-catenin signaling pathway was upregulated in xenograft tumors compared to the parental MKN45 cells (Supplementary Fig. 1A). We hypothesized that this might be the result of selective evolutionary pressure imposed by the tumor environment, favoring stress-resistant molecular traits. Concordant with this, it has recently been reported that metabolic stress induces stem-like phenoconversion through the WNT pathway (22). To confirm this, we generated metabolic-stress-selected MKN45 (sMKN45) cell lines as previously reported. Compared to parental MKN45 lines, sMKN45 cells showed a more aggressive tumor-forming phenotype (Supplementary Fig. 1B). Next, sMKN45 cells were subcutaneously injected into nude mice to establish xenografts. Treatment with anti-GPR177 antibody substantially reduced tumor progression in terms of both volume and weight (Fig. 4B). The sMKN45 cells showed increased GPR177 expression and sensitivity to anti-GPR177 antibody in vitro (Fig. 4C). Previous studies have clearly demonstrated that loss of GPR177 function in other types of cancer suppresses tumor formation in xenograft models $(8,13,18)$. Therefore, we tested whether GPR177 knockdown in GC cancer would inhibit tumor formation. Using shRNA targeting GPR177 (shGPR177), we generated AGS cell lines that stably express low levels of GPR177 mRNA and protein. Control (shCON) and stable shGPR177 AGS cells were subcutaneously injected into BALB/C nude mice ( $\mathrm{n}=5$ mice/group). Two weeks later, xenograft tumors were measured with a caliper every 2 days and terminated when volume exceeded $800 \mathrm{~mm}^{3}$. Compared to control cells, shGPR177-expressing AGS cells showed retarded growth rate (Fig. 4D). These data collectively show that targeted inhibition of GPR177 suppresses in vivo tumorigenesis of gastric cancer cells.

\section{Anti-GPR177 antibody treatment suppresses gastric tumorigenesis in PDX model}

Previously, we established GC patient-derived xenograft (PDX) model which could be applied to predict cancer patient's responses to the cancer drug (23). We tested whether this tumor suppression would be reproducible in a PDX model. Among GC patients who had undergone gastrectomy, GPR177 expression level of tumor specimens was determined by immunohistochemistry and designated high, moderate, low or negative (Fig. 4E and F). Case GA083, a GPR177 high expressing model, was expanded and divided randomly into two groups. One group was injected intravenously with anti-GPR177 monoclonal antibody \#3 every 2 days for 3 weeks, and the other group was injected with PBS as negative control. Tumor size was measured every 2 days (Fig. 4G). Both tumor size and weight were significantly reduced in antibody treatment group compared with control group (Fig. $4 \mathrm{G}$ and $\mathrm{H}$ ). Taken together, these data show that targeted inhibition of GPR177 suppresses human gastric tumorigenesis. 


\section{DISCUSSION}

GPR177 is seven-pass transmembrane protein that binds cytoplasmic WNT ligand and secretes it to the extracellular space (24). Dysregulation of WNT secretion and uncontrolled activation of the WNT/ $\beta$-catenin signaling pathway is associated with disease, such as cancer, and developmental abnormalities (25-29). GPR177 knockout results in WNT loss-of-function phenotypes in species ranging from Drosophila to Mus musculus. In cancer, GPR177 upregulation enhances tumor progression, and downregulation has been proposed as a potential therapeutic target.

Previous studies have shown that inhibition of GPR177 expression efficiently suppresses cancer progression (8, 13, 18). The cancer-promoting activity of GPR 177 has been explained in positive upregulation of the $\mathrm{WNT} / \beta$-catenin signal in most cancer types. In this study, we found that GPR177 is required in WNT/ $\beta$-catenin, signaling in GC cells.

We examined the role of GPR177 in the WNT-secreting pathway in GC cell lines. We expressed exogenous WNT1 ligand and confirmed that WNT was secreted into the culture medium. To prevent WNT secretion, we treated GC cells with anti-GPR177 monoclonal antibody. Because the antibodies were raised against the middle of the WNT-binding motif, we presumed that they would block this function of GPR177. Antibody treatment reduced not only WNT secretion but also cell viability and migration. As antibody treatment was effective only on WNT-secreting cells, we speculated that, whereas expression level is important for cancer cell proliferation, the presence of intact WNT-secretory machinery, including the ER, Golgi, and cargo carrier systems, was likely a more critical factor in the case of antibody treatment of GC. Thus, we concluded that GC cell lines that secrete WNT ligand are sensitive to monoclonal antibody treatment in vitro.

As a positive component of the WNT/ $\beta$-catenin signaling pathway, GPR177 is upregulated when the signal is activated. More precisely, $\beta$-catenin, which is stabilized and dissociated from the destructive complex, is translocated into the nucleus and transcriptionally activated. Consequently, GPR177 transcription is induced and mRNA expression increases. However, it is still not clear that in this mode of positive feedback regulation, a cell is induced to produce more WNT ligand in an autocrine manner. Regardless of whether WNT ligand synthesis is induced in a WNT-ligand-dependent or -independent manner, increased GPR177 expression is followed by induction of WNT/ $\beta$-catenin signaling.

Furthermore, we confirm that inhibition of GPR177 function effectively suppressed tumor progression in in vivo xenograft and PDX models. GC patients who are expressing high levels of GPR177 thereafter capable of WNT secretion could be tested whether the treatment of GPR177 antibody will suppress tumor progression. From clinical perspective, if we use the PDX model and personalized molecular information, we can stratify GC patients according to WNT-secretion proficiency and predict the therapeutic outcome of anti-GPR177 monoclonal antibody treatment as a novel therapy as described in Supplementary Fig. 2.

In conclusion, we report for the first time that GPR177 is highly expressed in GC patients and that GPR177 expression level is positively correlated with poor patient prognosis. Further, our findings provide a rationale of GPR177 inhibition as a therapeutic target for GC treatment.

\section{MATERIALS AND METHODS}

\section{Cell culture}

Cell lines were maintained in RPMI-1640 supplemented with $10 \%$ heat-inactivated fetal bovine serum and $1 \%$ penicillin/ streptomycin. 293FT and YCC cells were maintained in DMEM as previously described (30).

\section{Statistical analysis}

Prognosis was evaluated by overall survival, from day of surgery to death by any cause. Correlation of gene expression with overall survival was estimated using Kaplan-Meier survival plots and log-rank tests. Multivariable Cox proportional hazards regression analysis was used to evaluate independent prognostic factors associated with overall survival, including age, sex, Lauren type, and TNM-stage groups as covariates. A two-sided $\mathrm{P}$ value $<0.05$ was considered statistically significant. All statistical analyses were conducted in the $\mathrm{R}$ language environment (http://www.r-project.org).

\section{PDX model}

A PDX model was established as previously reported (23). Briefly, a tumor was obtained from a surgical specimen (F0) immediately after gastrectomy. Formation of a tumor greater than $500 \mathrm{~mm}^{3}$ in volume at the implant site was considered a successful engraftment in the case of subcutaneous implantation. The mouse was anaesthetized, and the tumor (F1) was removed for serial transplantation into the next generation (F2, F3). Mice were housed and fed according to the institutional guidelines for animal care. All animal experiments were approved by the Institutional Animal Care and Use Committee (IACUC) of the Yonsei University College of Medicine (2014-0130). Six-week old female athymic nude mice (Japan SLC, Inc., Japan) and male NOG mice (NOD/Shi-scid, IL-2 Rynull, Ciea, Japan) were used for each PDX model. Before the experiments, the animals were acclimated for seven days to a $12 \mathrm{~h}$ light/ $12 \mathrm{~h}$ dark cycle. One case of a GPR177-positive tumor specimen (F0) that was successfully maintained through passages was subcutaneously implanted into a NOG mouse (passage 1 or F1).

\section{ACKNOWLEDGEMENTS}

This study was supported by a grant of the Korean Health Technology R\&D Project, Ministry of Health \& Welfare, 
Republic of Korea (HI14C1840).

\section{CONFLICTS OF INTEREST}

The authors have no conflicting interests.

\section{REFERENCES}

1. Tan P and Yeoh KG (2015) Genetics and Molecular Pathogenesis of Gastric Adenocarcinoma. Gastroenterology 149, 1153-1162 e1153

2. Tan IB, Ivanova T, Lim KH et al (2011) Intrinsic subtypes of gastric cancer, based on gene expression pattern, predict survival and respond differently to chemotherapy. Gastroenterology 141, 476-485, 485.e471-411

3. Corso S and Giordano S (2016) How Can Gastric Cancer Molecular Profiling Guide Future Therapies? Trends Mol Med 22, 534-544

4. Bang Y-J, Van Cutsem E, Feyereislova A et al (2010) Trastuzumab in combination with chemotherapy versus chemotherapy alone for treatment of HER2-positive advanced gastric or gastro-oesophageal junction cancer (ToGA): a phase 3, open-label, randomised controlled trial. The Lancet 376, 687-697

5. Sartore-Bianchi A, Trusolino L, Martino C et al (2016) Dual-targeted therapy with trastuzumab and lapatinib in treatment-refractory, KRAS codon 12/13 wild-type, HER2-positive metastatic colorectal cancer (HERACLES): a proof-of-concept, multicentre, open-label, phase 2 trial. Lancet Oncol 17, 738-746

6. Wilke H, Muro K, Van Cutsem E et al (2014) Ramucirumab plus paclitaxel versus placebo plus paclitaxel in patients with previously treated advanced gastric or gastrooesophageal junction adenocarcinoma (RAINBOW): a double-blind, randomised phase 3 trial. Lancet Oncol 15, 1224-1235

7. Kahn M (2014) Can we safely target the WNT pathway? Nat Rev Drug Discov 13, 513-532

8. Lu D, Li Y, Liu QR et al (2015) Wls promotes the proliferation of breast cancer cells via Wnt signaling. Med Oncol 32, 140

9. Mao J, Fan S, Ma W et al (2014) Roles of Wnt/betacatenin signaling in the gastric cancer stem cells proliferation and salinomycin treatment. Cell Death Dis 5, e1039

10. Clevers $\mathrm{H}$ and Nusse $\mathrm{R}$ (2012) Wnt/beta-catenin signaling and disease. Cell 149, 1192-1205

11. Anastas JN and Moon RT (2013) WNT signalling pathways as therapeutic targets in cancer. Nat Rev Cancer $13,11-26$

12. Proffitt KD and Virshup DM (2012) Precise regulation of porcupine activity is required for physiological Wnt signaling. J Biol Chem 287, 34167-34178

13. Voloshanenko O, Erdmann G, Dubash TD et al (2013) Wnt secretion is required to maintain high levels of Wnt activity in colon cancer cells. Nat Commun 4, 2610

14. Banziger C, Soldini D, Schutt C, Zipperlen P, Hausmann $\mathrm{G}$ and Basler K (2006) Wntless, a conserved membrane protein dedicated to the secretion of Wnt proteins from signaling cells. Cell 125, 509-522

15. Bartscherer $K$, Pelte $N$, Ingelfinger $D$ and Boutros $M$ (2006) Secretion of Wnt ligands requires Evi, a conserved transmembrane protein. Cell 125, 523-533

16. Yu J, Chia J, Canning CA, Jones CM, Bard FA and Virshup DM (2014) WLS retrograde transport to the endoplasmic reticulum during Wnt secretion. Dev Cell 29, 277-291

17. Maruyama EO, Yu HM, Jiang M, Fu J and Hsu W (2013) Gpr177 deficiency impairs mammary development and prohibits Wnt-induced tumorigenesis. PLoS One 8, e56644

18. Augustin I, Goidts V, Bongers A et al (2012) The Wnt secretion protein Evi/Gpr177 promotes glioma tumourigenesis. EMBO Mol Med 4, 38-51

19. Barrott J, Cash GM, Smith AP, Barrow JR and Murtaugh LC (2011) Deletion of mouse Porcn blocks Wnt ligand secretion and reveals an ectodermal etiology of human focal dermal hypoplasia/Goltz syndrome. Proc Natl Acad Sci U S A 108, 12752-12757

20. Liu J, Pan S, Hsieh MH et al (2013) Targeting Wnt-driven cancer through the inhibition of Porcupine by LGK974. Proc Natl Acad Sci U S A 110, 20224-20229

21. Minn YK, Lee DH, Hyung WJ et al (2014) MicroRNA-200 family members and ZEB2 are associated with brain metastasis in gastric adenocarcinoma. Int J Oncol 45, 2403-2410

22. Lee E, Yang J, Ku M et al (2015) Metabolic stress induces a Wnt-dependent cancer stem cell-like state transition. Cell Death Dis 6, e1805

23. Choi YY, Lee JE, Kim H et al (2016) Establishment and characterisation of patient-derived xenografts as paraclinical models for gastric cancer. Sci Rep 6, 22172

24. Kim W, Kim M and Jho EH (2013) Wnt/beta-catenin signalling: from plasma membrane to nucleus. Biochem J 450, 9-21

25. Fu J, Ivy Yu HM, Maruyama T, Mirando AJ and Hsu W (2011) Gpr177/mouse Wntless is essential for Wnt-mediated craniofacial and brain development. Dev Dyn 240, 365-371

26. Jin J, Morse M, Frey C, Petko J and Levenson R (2010) Expression of GPR177 (Wntless/Evi/Sprinter), a highly conserved Wnt-transport protein, in rat tissues, zebrafish embryos, and cultured human cells. Dev Dyn 239, 2426-2434

27. $\mathrm{Yu}$ HM, Jin $\mathrm{Y}, \mathrm{Fu} J$ and Hsu W (2010) Expression of Gpr177, a Wnt trafficking regulator, in mouse embryogenesis. Dev Dyn 239, 2102-2109

28. Carpenter AC, Smith AN, Wagner H et al (2015) Wnt ligands from the embryonic surface ectoderm regulate 'bimetallic strip' optic cup morphogenesis in mouse. Development 142, 972-982

29. Jiang M, Ku WY, Fu J, Offermanns S, Hsu W and Que J (2013) Gpr177 regulates pulmonary vasculature development. Development 140, 3589-3594

30. Park SY, Seo J, Choi HK et al (2017) Protein serine/ threonine phosphatase PPEF-1 suppresses genotoxic stress response via dephosphorylation of PDCD5. Sci Rep 7, 39222 